J. Sustain. Wireless Syst.

Vol.01/ No. 01

Pages: 19-30

http://irojournals.com/irosws/

DOI: https://doi.org/10.36548/jsws.2019.1.002

\title{
TOWARDS GHZ METALLIC ACCESS NETWORKS
}

Petr Jares,

Professor,

Czech Technical University in Prague, Faculty of Electrical Eng., Department of Telecommunication

Engineering, Technicka, Prague, Czech Republic

Email: petr.jares@fel.cvut.cz

Pavel Lafata,

Professor,

Czech Technical University in Prague, Faculty of Electrical Eng., Department of Telecommunication Engineering, Technicka, Prague, Czech Republic

Email: pavel.lafata@fel.cvut.cz

\begin{abstract}
The transmission capacity and speed of access networks need to be continually increased as the transmission demands and data flows of the end-users grow steadily. Metallic cables and lines are still often used in both local area networks as well as access networks. Today, the G.fast lines with transmission speed of $1 \mathrm{Gbps}$ working in $212 \mathrm{MHz}$ frequency band are being deployed in practice, and a novel XG.fast conception with a frequency band up to $424 \mathrm{MHz}$ is being developed. However, the future DSL solutions will soon reach GHz transmission bands in order to increase the transmission speed over metallic lines. Due to that it is necessary to investigate the behaviour and transmission characteristics of typical existing metallic lines in $\mathrm{GHz}$ frequency band and to develop accurate models to provide necessary estimations and modelling of in order to optimize transmission parameters, modulations and characteristics of future gigabit DSL solutions.
\end{abstract}

Keywords: G.fast; Gigabit DSL; metallic cables; modelling.

\section{Introduction}

Today, there are numerous access networks solutions including various types of wireless networks [1], [2], optical networks [3] and metallic lines used in practice [4]. The metallic cables and lines are still often used in various types of local area networks, access networks and premises network solutions [5]. Moreover, their combination with optical networks in various types of FTTx (Fiber to the X) lines is possible [6], [7]. The main advantage of these 
J. Sustain. Wireless Syst.

Vol.01/ No. 01

Pages: 19-30

http://irojournals.com/irosws/

DOI: https://doi.org/10.36548/jsws.2019.1.002

solutions is that the exploitation of existing metallic lines can potentially decrease the capital expenditures (CAPEX) of newly deployed networks [8]. In order to provide modern metallic subscriber lines solutions, G.fast and Gigabit DSL with gigabit transmission speed was developed [9]. These solutions use wide transmission frequency band in order to achieve Gbps speed, G.fast lines typically occupy frequency band up to 106 or $212 \mathrm{MHz}$. The future development is now focused on upgrading the transmission speed and capacity of metallic lines and to occupy the frequency band up to GHz. The upgraded XG.fast line technology will probably exploit frequency band up to $424 \mathrm{MHz}$ [10]. Another way of the progress is Terabit DSL conception, which has been recently presented in [11].

On the other hand, the transmission parameters of typical existing metallic cables and lines strongly depend on the frequency band. Especially the attenuation as well as phase characteristics are affected by the frequency band and due to that, the transmission speed of DSL strongly depends on the frequency band as well as on the length of the metallic line section [12]. There are also several negative phenomena, which negatively influence the resulting transmission capacity, especially crosstalk (far-end crosstalk, FEXT, near-end crosstalk, NEXT) plays the major role in multi-quad and multi-pair metallic cables [13]. In order to predict, analyse and model these transmission parameters of metallic lines and metallic cables, extensive measurements and modelling is necessary. By developing accurate models, which can predict transmission parameters of typical existing lines, the optimization of transmission parameters, such as modulation formats, crosstalk reduction techniques, transmitter and receiver signal parameters, can be performed in order to increase the overall performance of the networks [12].

This paper contains the results of experimentations and measurements of several types of metallic cables often used and deployed in access network segments. The measurements were performed in a frequency band up to $1.2 \mathrm{GHz}$ and both components of a propagation constant $\square$ (f), an attenuation constant $\square$ (f) and a phase constant $\square$ (f), were measured. Next, several existing models were used to determine their accuracy of modelling these transmission characteristics up to $1.2 \mathrm{GHz}$. Finally, the resulting parameters were used to estimate the transmission performance of future XG.fast conception with frequency band up to $424 \mathrm{MHz}$. 
J. Sustain. Wireless Syst.

Vol.01/ No. 01

Pages: 19-30

http://irojournals.com/irosws/

DOI: https://doi.org/10.36548/jsws.2019.1.002

These estimated results of XG.fast performance for different types of metallic cables and lengths of lines together with the conclusion are presented in the last section of this article.

\section{Related Works}

Today, there are several different types of models used to estimate the transmission characteristics of metallic lines [14]. However, most of them were defined for $\mathrm{MHz}$ frequency band and their accuracy up to $1 \mathrm{GHz}$ should be investigated. Moreover, it is necessary to perform the measurements of various types of typical metallic cables up to 1 $\mathrm{GHz}$ first, as these measurements and experiments are missing.

\subsection{Existing Transmission Line Models}

A group of British Telecom models was created to approximate the primary line coefficients, $R(f), L(f), C(f)$ and $G(f)$ of various types of metallic lines and cables [15]. The full version uses 13 parameters in summary specified for each type of metallic line individually. Nevertheless, the BT models were originally derived and created for DSL applications up to $100 \mathrm{MHz}$ and although they are often used for higher frequency bands, their accuracy for hundreds of $\mathrm{MHz}$ or up to $1 \mathrm{GHz}$ applications is questionable.

The ITU-T presented a novel transmission line model together with new G.fast generation of transmission lines within the ITU-T G.9701 recommendation [16], [17]. This model is used as a reference model suited for the purpose of G.fast simulations and estimations. The G.fast model is based on modelling of longitudinal impedance $Z_{S}(j \omega)$ and shunt admittance $Y_{P}(j \omega)$ and it includes various high-frequency effects, such as the skin effect, proximity effect, etc. However, the model requires 9 specific parameters for each type of metallic cable and together with its complexity, this makes the application of the model in practice difficult.

Today, the Chen's model together with a group of KM models (KM1, KM2 and KM3) are the most frequently used models for approximations of metallic lines [14]. Chen in [18] proposed a simple model for approximations of the attenuation constant $\alpha(f)$ as (1): 
J. Sustain. Wireless Syst.

Vol.01/ No. 01

Pages: $19-30$

http://irojournals.com/irosws/

DOI: https://doi.org/10.36548/jsws.2019.1.002

$$
\alpha(f)=k_{1} \cdot \sqrt{f}+k_{2} \cdot f \quad[\mathrm{~dB} / \mathrm{km} ; \mathrm{Hz}],
$$

where $k_{1}$ and $k_{2}$ are specific parameters for each metallic line (cable). The accuracy of the model was also validated in [14], in which the causality of Chen's model was corrected and the resulting model was referred as KM1. The phase constant, $\beta(f)$, is in the KM1 model approximated as (2):

$$
\beta(f)=k_{1} \cdot \sqrt{f}-\frac{2}{\pi} k_{2} \cdot f \cdot \ln (f)+k_{3} \cdot f \quad[\mathrm{rad} / \mathrm{km} ; \mathrm{Hz}],
$$

in which $k_{1}$ and $k_{2}$ parameters are the same ones, as already used in (1), and $k_{3}$ is newly specified for each metallic line. Further improvements by applying the Hilbert transform into KM1 model by Acatauassu et al. led into introducing KM2 and KM3 models in [14], both containing more $k$-parameters and providing better accuracy. The KM2 model is in [14] specified as (3):

$$
\begin{aligned}
& \alpha(f)=k_{1} \cdot \sqrt{f}+k_{2} \cdot f+k_{4} \quad[\mathrm{~dB} / \mathrm{km} ; \mathrm{Hz}], \\
& \beta(f)=k_{1} \cdot \sqrt{f}-\frac{2}{\pi} k_{2} \cdot f \cdot \ln (f)+k_{3} \cdot f \quad[\mathrm{rad} / \mathrm{km} ; \mathrm{Hz}]
\end{aligned} .
$$

One extra term with a $k_{4}$ parameter was added for modeling of an attenuation constant $\alpha(f)$, while the model for $\beta(f)$ is completely the same as in KM1 model. Finally, the KM3 model presented again in [26] is expressed as (4):

$$
\begin{aligned}
& \alpha(f)=k_{1} \cdot \sqrt{f}+k_{2} \cdot f+k_{5} \cdot \frac{1}{\sqrt{f}}+k_{4} \quad[\mathrm{~dB} / \mathrm{km} ; \mathrm{Hz}], \\
& \beta(f)=k_{1} \cdot \sqrt{f}-\frac{2}{\pi} k_{2} \cdot f \cdot \ln (f)+k_{3} \cdot f-k_{5} \cdot \frac{1}{\sqrt{f}} \quad[\mathrm{rad} / \mathrm{km} ; \mathrm{Hz}]
\end{aligned}
$$

Another term with a $k_{5}$ parameter was added, therefore the KM3 model contains $5 k$ parameters in summary. According to the results presented in [14], the proposed KM1, KM2, KM3 models are suitable for frequencies of hundreds of MHz.

A completely different approach and modelling is used in Inverse Hyperbolic Sine Model, Arsinh model, presented by Lafata in [19]. By extensive mathematical derivation and various experimentation with real metallic cable, Lafata discovered that both components of a propagation constant, $\chi(f)$, an attenuation constant $\alpha(f)$ as well as a phase constant $\beta(f)$, can be 
J. Sustain. Wireless Syst.

Vol.01/ No. 01

Pages: $19-30$

http://irojournals.com/irosws/

DOI: https://doi.org/10.36548/jsws.2019.1.002

approximated using inverse hyperbolic sine function. Afterwards, using Taylor series expansion and several assumptions and substitutions, the Arsinh model was proposed as (5), (6):

$$
\begin{aligned}
& \alpha(f)=k_{1} \cdot \operatorname{arsinh}\left(k_{2} \cdot f^{0.4}+k_{3} \cdot f^{k_{4}}\right) \quad[\mathrm{dB} / \mathrm{km}], \\
& \beta(f)=k_{5} \cdot f-\operatorname{arsinh}\left(k_{2} \cdot f\right) \quad[\mathrm{rad} / \mathrm{km}] .
\end{aligned}
$$

Evidently, the Arsinh model uses $5 k$-parameters, the same number of $k$-parameters as used in the KM3 model, however, its accuracy according to the results presented in [19] is slightly better.

\section{Experimental Results}

This section contains the results of performed experimental measurements and modelling of several typical types of metallic cables. Next, these results were adopted and used for estimation of Gigabit DSL application in order to analyse the transmission potential of future DSL systems operating in a frequency band of hundreds of MHz.

\subsection{Measurements and Modelling of Metallic Cables up to $1.2 \mathrm{GHz}$}

First, extensive measurements of several different types of typical metallic access cables were performed. In order to analyse their transmission performance for future Gigabit DSL applications, these cables were measured in a frequency band up to $1.2 \mathrm{GHz}$. The purpose of these measurements was to examine the accuracy of existing metallic lines models described in the previous section in order to identify the most accurate and suitable model for further estimations. While all these models in section 2.1 were tested in a frequency band of hundreds of $\mathrm{MHz}$, their accuracy up to $1.2 \mathrm{GHz}$ should be investigated first.

In order to cover as many different types of metallic cables as possible, the measurements were performed for following samples of metallic cables: typical structured indoor cabling systems - UTP cat. 5e (21.5 meters), UTP cat. 6 (55.7 meters), SYKFY $4 \times 2 \times 0.5$ (25.4 meters) and UCEKFY $4 \times 2 \times 0.5$ (105 meters). All measurements were performed using calibrated Rohde\&Schwarz vector network analyser together with North Hill High Frequency Balun NH16447 up to $1.2 \mathrm{GHz}$, the measurement error was calculated and properly calibrated throughout the entire measurements [19]. All metallic cables were measured in a frequency band between 5 and $1200 \mathrm{MHz}$ in order to compare all models at 
J. Sustain. Wireless Syst.

Vol.01/ No. 01

Pages: $19-30$

http://irojournals.com/irosws/

DOI: https://doi.org/10.36548/jsws.2019.1.002

future Gigabit DSL frequencies. All attenuation and phase constants obtained either by measurements or through all models were recalculated for the standardized length $l=1$ meter.

The first comparison was performed for UTP cat. 5e cable. Since this type of metallic cable contains 4 twisted pairs, the measurements were performed for all of them, however, the following Fig. 1 contains the attenuation constant $\alpha(f)$ and Fig. 2 the phase constant $\beta(f)$ for only one selected twisted pair. Both figures contain the comparison between measured result as well as KM1, KM3 and Arsinh model.

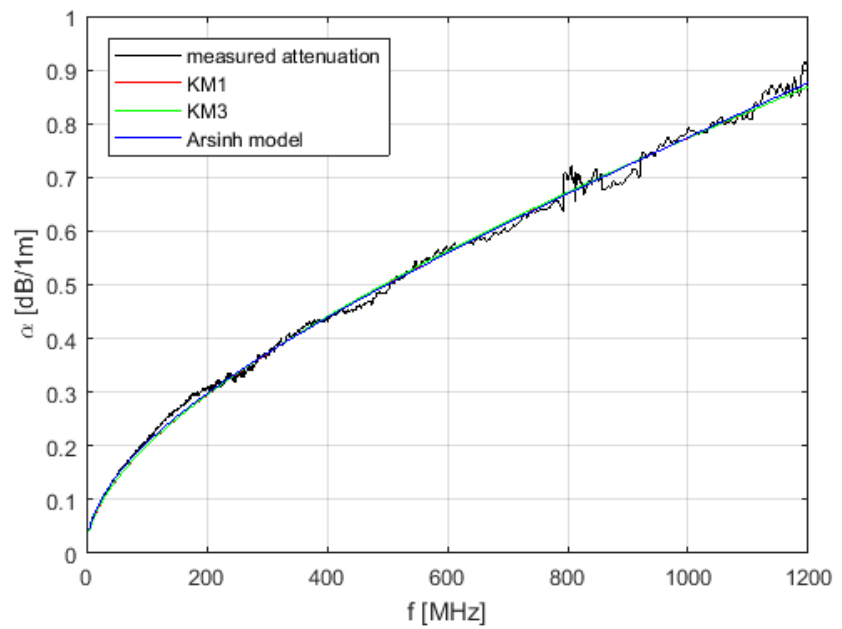

Fig. 1. Measurement and modelling of attenuation constant $\alpha(f)$ of UTP cat. 5e cable. 
J. Sustain. Wireless Syst.

Vol.01/ No. 01

Pages: $19-30$

http://irojournals.com/irosws/

DOI: https://doi.org/10.36548/jsws.2019.1.002

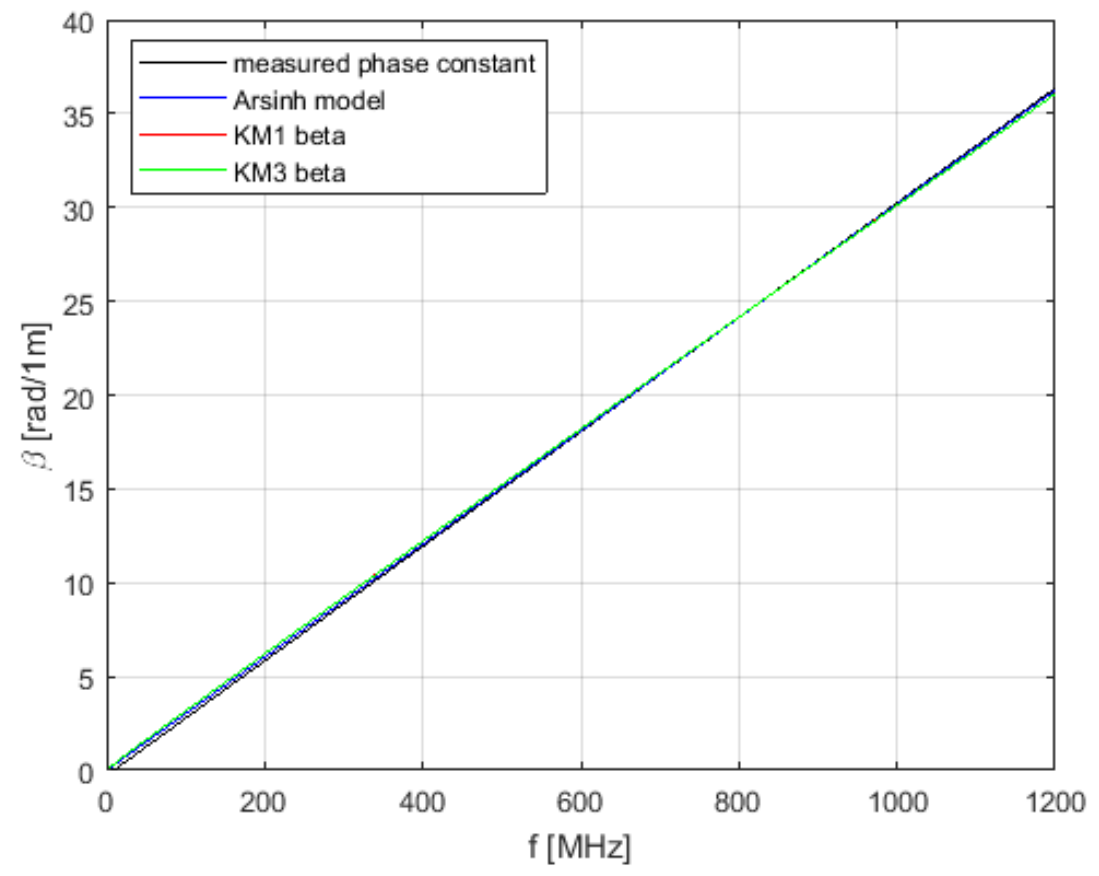

Fig. 2. Measurement and modelling of phase constant $\beta(f)$ of UTP cat. 5e cable.

Evidently, the UTP cat. 5e cable was able to operate in the frequency band up to $1.2 \mathrm{GHz}$ and the cable is suitable for Gigabit DSL applications. In order to compare the accuracy of all models, the squared error sum $E_{X}$ through the entire frequency band was calculated for both attenuation constant $\alpha(f)$ as well as phase constant $\beta(f)$ according to [19] as (7):

$$
E_{X}=\sum_{f}\left(X_{M}(f)-X(f)\right)^{2} .
$$

In (7), the summary squared error $E_{X}$ is calculated for modeled characteristic $(\alpha(f)$, $\beta(f))$ as the sum of squared differences between modeled characteristic values $X_{M}(f)$ and real measured values $X(f)$ at each frequency step $f$.

Tab. 1. Summary squared errors calculated for both $\alpha(f), \beta(f)$ constants of each model for UTP cat. 5e cable.

\begin{tabular}{|c|c|c|c|}
\hline $\begin{array}{c}\text { Summary squared } \\
\text { error } E_{X}\end{array}$ & KM1 model & KM3 model & Arsinh model \\
\hline$\alpha(f)[\mathrm{dB} / 1 \mathrm{~m}]$ & 1.68474 & 1.68404 & 1.62750 \\
\hline$\beta(f)[\mathrm{rad} / 1 \mathrm{~m}]$ & 381.42537 & 381.65179 & 99.42537 \\
\hline
\end{tabular}


J. Sustain. Wireless Syst.

Vol.01/ No. 01

Pages: $19-30$

http://irojournals.com/irosws/

DOI: https://doi.org/10.36548/jsws.2019.1.002

It is evident that the Arsinh model provides the most accurate approximation of both transmission characteristics. Generally, all three models are suitable for frequency band up to $1.2 \mathrm{GHz}$.

The same experimentation and modeling was performed for SYKFY $4 \times 2 \times 0.5$ cable as well. The result of measurement of attenuation constant $\alpha(f)$ as well as phase constant $\beta(f)$ in a frequency band $1.2 \mathrm{GHz}$ for SYKFY cable is illustrated in Fig. 3 and Fig. 4.

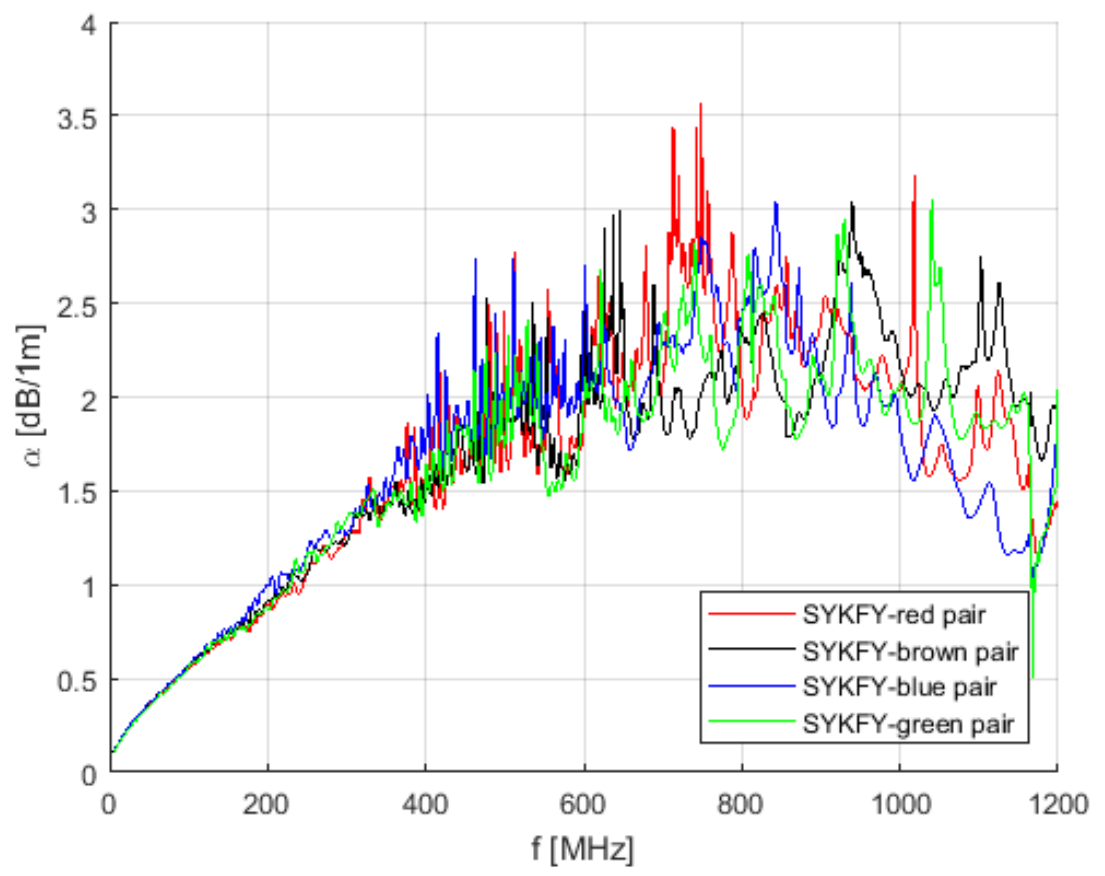

Fig. 3. Measured characteristic of attenuation constant $\alpha(f)$ of SYKFY cable. 
J. Sustain. Wireless Syst.

Vol.01/ No. 01

Pages: $19-30$

http://irojournals.com/irosws/

DOI: https://doi.org/10.36548/jsws.2019.1.002

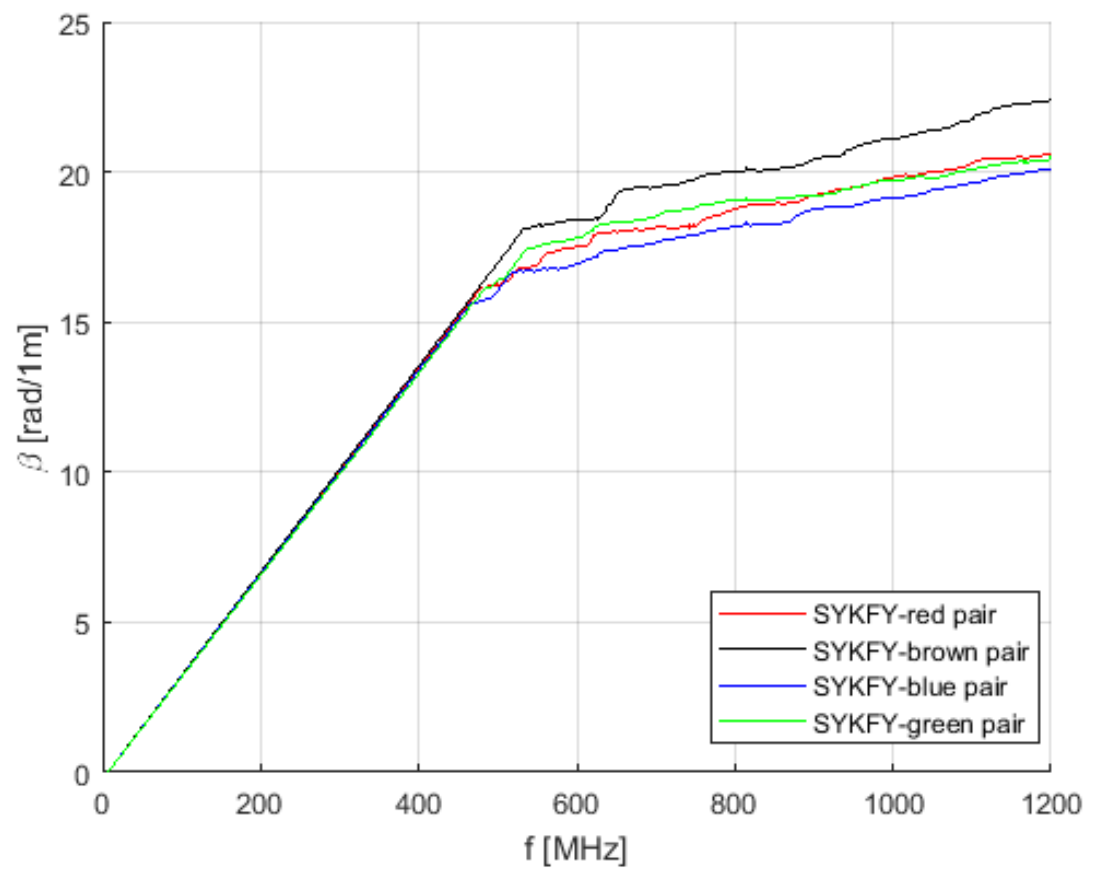

Fig. 4. Measured characteristic of phase constant $\beta(f)$ of SYKFY cable.

It is obvious that the SYKFY cable is not suitable for frequencies above approx. 450 $\mathrm{MHz}$, as the character of its attenuation constant $\alpha(f)$ as well as phase constant $\beta(f)$ differentiate. Evidently, the signals propagating throughout the pairs emits their energy among all surrounding pairs, therefore the crosstalk becomes dominant propagation limitation. Due to that, all the following processing of SYKFY cable type is performed only up to $450 \mathrm{MHz}$. Similarly to SYKFY, the same problem can be observed in case of UCEKFY cable. The frequency characteristics of UCEKFY cable were therefore limited only up to 120 $\mathrm{MHz}$, while the transmission parameters of UCEKFY cable above this frequency band are not suitable for Gigabit DSL application. The results of summary squared error for SYKFY, UCEKFY and UTP cat. 6 cable are presented in Table 2.

Tab. 2. Summary squared errors calculated for both $\alpha(f), \beta(f)$ constants of each model for UTP cat. 5e cable. 
J. Sustain. Wireless Syst.

Vol.01/ No. 01

Pages: $19-30$

http://irojournals.com/irosws/

DOI: https://doi.org/10.36548/jsws.2019.1.002

\begin{tabular}{|c|c|c|c|}
\hline $\begin{array}{c}\text { Summary squared } \\
\text { error } E_{X}\end{array}$ & KM1 model & KM3 model & Arsinh model \\
\hline \multicolumn{4}{|c|}{ UTP cat. 6 cable } \\
\hline$\alpha(f)[\mathrm{dB} / 1 \mathrm{~m}]$ & 4.85074 & 4.85040 & 4.89255 \\
\hline$\beta(f)[\mathrm{rad} / 1 \mathrm{~m}]$ & 152.03886 & 152.49982 & 8.44084 \\
\hline \multicolumn{4}{|c|}{ SYKFY cable } \\
\hline$\alpha(f)[\mathrm{dB} / 1 \mathrm{~m}]$ & 9.57910 & 9.57828 & 9.54887 \\
\hline$\beta(f)[\mathrm{rad} / 1 \mathrm{~m}]$ & 250.78287 & 252.04988 & 252.04988 \\
\hline \multicolumn{4}{|c|}{$\mathrm{UCEKFY} \mathrm{cable}$} \\
\hline$\alpha(f)[\mathrm{dB} / 1 \mathrm{~m}]$ & 0.15644 & 0.15610 & 0.12924 \\
\hline$\beta(f)[\mathrm{rad} / 1 \mathrm{~m}]$ & 13.54425 & 15.82561 & 5.78733 \\
\hline
\end{tabular}

According to the results in Tab. 2, the Arsinh model performs usually the most accurate estimations of all metallic cables, however, in some cases, the KM3 is better. Generally, the differences between the results of all three models are not significant.

\subsection{Modelling of XG.fast Performance}

In this section, the results of XG.fast performance are presented. The estimations are performed for all 4 types of metallic cables and their transmission characteristics presented in previous section. According to the ITU-T documents and presentations [16], [17], the subchannel spacing of XG.fast DMT carriers is currently being considered as $f c=51.75 \mathrm{kHz}$ [10], the starting frequency considered for XG.fast system is $2 \mathrm{MHz}$, while the upper frequency used in the simulations was $120 \mathrm{MHz}$ for UCEKFY cable and $424 \mathrm{MHz}$ for the rest of the cables, given as the number of XG.fast subchannels $n=8192$ and the subchannel spacing $51.75 \mathrm{kHz}$ [10]. No band notching was considered except the first 40 subchannels were masked ( 0 bit allocation) in accordance to the ITU-T documents [16], [17]. The power spectral density PSD transmission mask in all performed simulations is a constant value of $S(n)=-65 \mathrm{dBm} / \mathrm{Hz}$ up to $30 \mathrm{MHz},-73 \mathrm{dBm} / \mathrm{Hz}$ at $30 \mathrm{MHz}$ and linearly decreasing towards $79 \mathrm{dBm} / \mathrm{Hz}$ at $424 \mathrm{MHz}$. The typical power spectral density of AWGN noise (Additive White Gaussian Noise) is $\sigma^{2}=-140 \mathrm{dBm} / \mathrm{Hz}$ [19]. The Shannon gap capacity used for calculations 
J. Sustain. Wireless Syst.

Vol.01/ No. 01

Pages: $19-30$

http://irojournals.com/irosws/

DOI: https://doi.org/10.36548/jsws.2019.1.002

is $\Gamma=10.75 \mathrm{~dB}$, which is a typical value for today's DSL and upcoming XG.fast systems [19]. Due to the high amount of sub-carriers and limits of $\mathrm{AD}$ and $\mathrm{DA}$ converters, the maximum bits loaded per QAM constellation in XG.fast was selected as bmax $=12$ bits [10]. All following XG.fast performance evaluations are based on standard formula for calculating DSL summary data rate $P$ for a channel, which can be expressed according to [19] as (8):

$$
P=f_{c} \sum_{n=1}^{N}\left\lfloor\min \left(\log _{2}\left(1+\frac{S(n) \cdot|H(n, l)|^{2}}{\Gamma \cdot\left(\sigma^{2}\right)}\right), b_{\max }\right)\right][\mathrm{bps}] .
$$

Where $\left|H_{i}(n)\right|^{2}$ is the power transfer function of a channel, which can be obtained from measured attenuation characteristics. The summation is performed for all $n$ frequency channels. The resulting performance of XG.fast was calculated as a function of length of a cable 1 for all 4 types of metallic cables. The result is presented in Fig. 5.

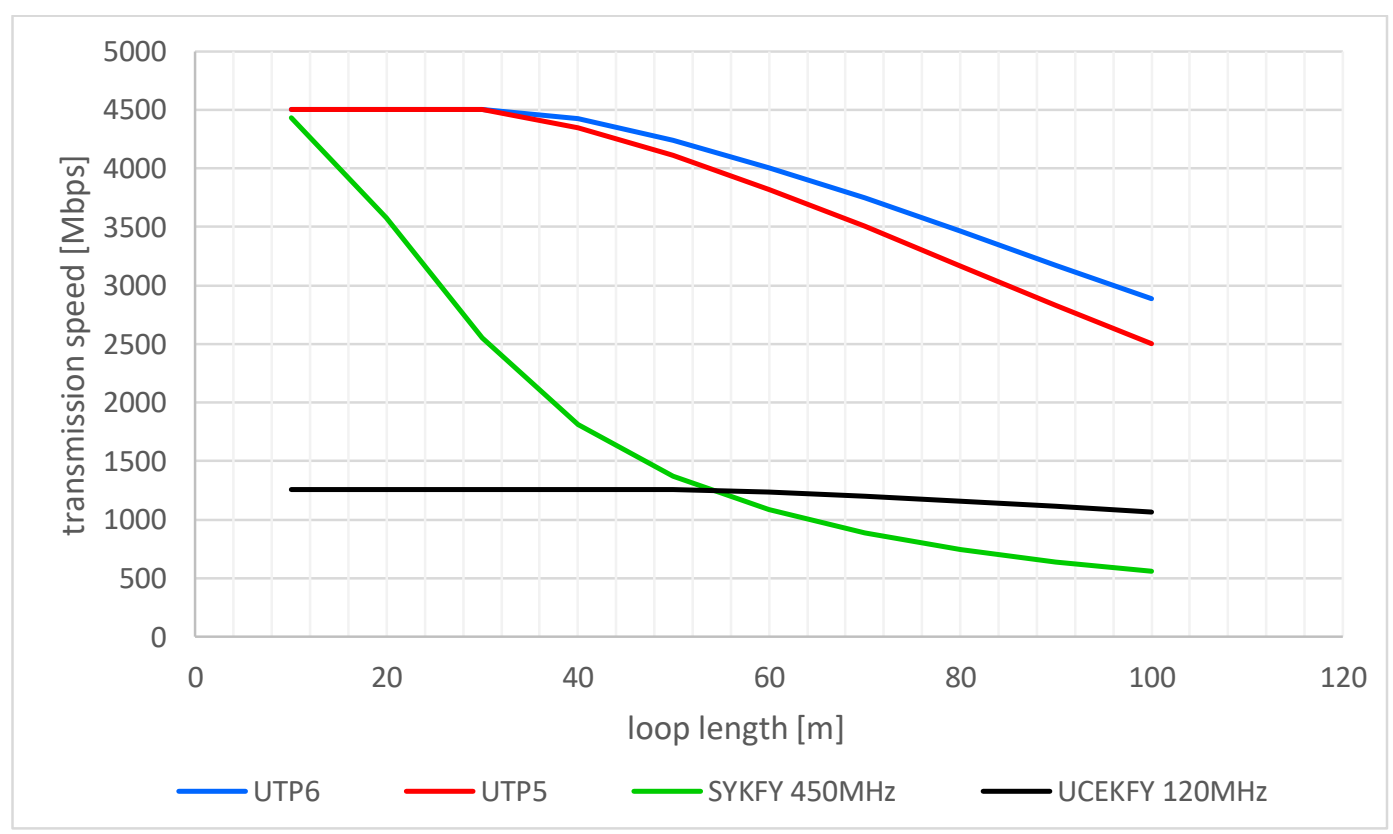

Fig. 5. The estimated summary transmission speed for all 4 types of cables and length $l$.

It is evident that the best performance provides the UTP cat. 6 cable, the estimated transmission speed for UTP cat. 5e is only slightly lower. Both SYKFY and UCEKFY cables are not suitable for Gigabit DSL applications, as their performance is significantly lower. 
J. Sustain. Wireless Syst.

Vol.01/ No. 01

Pages: 19-30

http://irojournals.com/irosws/

DOI: https://doi.org/10.36548/jsws.2019.1.002

This is mainly due to their frequency limitations and transmission problems at higher frequencies.

\section{Conclusions}

In this article, we performed experimentation, measurement and estimations of transmission parameters of 4 different types of metallic cables used in access networks. We measured the attenuation factor $\alpha(f)$ as well as phase factor $\beta(f)$ of UTP cat. 5e, UTP cat. 6, SYKFY and UCEKFY cables in a frequency band up to $1.2 \mathrm{GHz}$ in order to analyse the transmission parameters of these cables for their potential application for modern Gigabit DSL systems. We also compared the accuracy of 3 different models, KM1, KM3 and Arsinh model. Generally, all models provided similar results and are suitable for frequencies up to 1.2 GHz for all 4 types of metallic cables. The Arsinh model provided slightly more accurate results, especially for modelling of phase constant $\beta(f)$. Next, we used the measured and modelled transmission characteristics of all 4 metallic cables to estimate the transmission performance of modern Gigabit DSL, XG.fast lines. According to these estimations, the XG.fast line can reach gigabits transmission speed based on the length of a metallic loop and the type of a metallic cable. The transmission performance for both UTP cables were significantly better compared to SYKFY and CEKFY cables, as expected.

\section{References}

[1] Samanta, S. K. and Chanda, C. K. Wireless power network design through smart grid transmission system model. In International Conference on Energy, Power and Environment: Towards Sustainable Growth, 2015 (pp. 1-5). ICEPE.

[2] Proskochylo, A., Vorobyov, A., Zriakhov, M., Kravchuk, A., Akulynichev, A. and Lukin, V. Overview of wireless technologies for organizing sensor networks. In Second International ScientificPractical Conference Problems of Infocommunications Science and Technology, 2015 (pp. 39-41). PIC S\&T.

[3] Terada, J., Shimada, T., and Otaka, A. Optical access network technologies for future radio access networks.In IEEE Photonics Society Summer Topical Meeting Series, 2017 (pp. 37-38). SUM. 
J. Sustain. Wireless Syst.

Vol.01/ No. 01

Pages: 19-30

http://irojournals.com/irosws/

DOI: https://doi.org/10.36548/jsws.2019.1.002

[4] El-Ghazali Hamza, M. and Bashir Bugaje, K. Enhancement of Gigabit Passive Optical Highspeed Network using Fiber-To-The-Home. In International Conference on Computer, Control, Electrical, and Electronics Engineering, 2018 (pp. 1-4). ICCCEEE.

[5] Oksman, V. et al., 2016. The ITU-T's new G.fast standard brings DSL into the gigabit era. IEEE Communications Magazine, 54(3), pp. 118-126.

[6] Kiycioğlu, D. and Ünverdi, N. Ö. Applications of FTTX technology in optical communication systems. In 26th Signal Processing and Communications Applications Conference, 2018 (pp. 1-4). SIU.

[7] Araújo, M., Ekenberg, L. and Confraria, J. Rural networks cost comparison between 5G (mobile) and FTTx (fixed) scenarios. In IEEE 29th Annual International Symposium on Personal, Indoor and Mobile Radio Communications, 2018 (pp. 259-264). PIMRC.

[8] Medlen, N., Gilfedder, T. and Rafel, A., 2012. Novel infrastructure network design approach to support resilience in FTTx deployment. IEEE Communications Magazine, 50, pp. 114-120.

[9] Henao, D. H., Louveaux, J. and Maierbacher, G. Towards a Range-Enhanced and SpectrumFriendly G.fast. In IEEE Global Communications Conference, 2016 (pp. 1-7). GLOBECOM.

[10] Coomans, W. et al., 2015. XG-fast: the 5th generation broadband. IEEE Communications Magazine, 53(12), pp. 83-88.

[11] Cioffi, J. M., Kerpez, K. J., Hwang, C. S. and Kanellakopoulos, I., 2018. Terabit DSLs. IEEE Communications Magazine, 56(11), pp. 152-159.

[12] Timmers, M., Guenach, M., Nuzman, C. and Maes, J., 2013. G.fast: evolving the copper access network. IEEE Communications Magazine, 51, pp. 74-79.

[13] Leung, C., Huberman, S., Ho-Van, K., Le-Ngoc, T., 2013. Vectored DSL: Potential, Implementation Issues and Challenges. IEEE Communications Surveys \& Tutorials, 15, pp. 19071923.

[14] Acatauassu, D., Host, S., Chenguang, L., Berg, M., Klautau, A. and Borjesson, P. O., 2014. Simple and Causal Copper Cable Model Suitable for G.fast Frequencies. IEEE Transactions on Communications, 62, pp. 4040-4051.

[15] Hughes, H., 1997. Telecommunications Cables: Design, Manufacture and Installation. John Wiley\&Sons Ltd.

[16] ITU-T, 2014. G.9700 - Fast access to subscriber terminals (G.fast) - Power spectral density specification. ITU-T recommendation, Series G: Transmission Systems and Media, Digital Systems and Networks. 
J. Sustain. Wireless Syst.

Vol.01/ No. 01

Pages: $19-30$

http://irojournals.com/irosws/

DOI: https://doi.org/10.36548/jsws.2019.1.002

[17] ITU-T, 2014. G.9701 - Fast access to subscriber terminals (G.fast) - Physical layer specification ITU-T recommendation, Series G: Transmission Systems and Media, Digital Systems and Networks.

[18] Chen, W. Y., 1998. DSL: Simulation Techniques and Standards Development for Digital Subscriber Line System. Macmillan Technology Series.

[19] Lafata, P., 2017. Accurate low complexity modeling of twisted pairs suitable for G.fast frequencies. International Journal of Communication Systems, 30(6), pp. 1-16. 